\title{
Percepciones de profesores sobre factores que limitan la formación investigativa en estudiantes universitarios del área de Ciencias Sociales y Humanidades
}

\author{
Perceptions of professors on factors that limit research training in university students in the area of \\ Social Sciences and Humanities \\ Jacinta Hernández-Pérez ${ }^{a}$
}

\begin{abstract}
:
The study analysed from a comparative perspective, the perceptions of the professors of the area of Social Sciences and Humanities of two public universities: one Mexican and one Colombian, on the factors that limit the formation in scientific research of students of the Bachelor of Education. It was descriptive and cross-sectional. Twenty one teachers: eleven professors from the Mexican university and ten from the Colombian university were interviewed. Nine factors were compared with the Bereday's Model. The results reveal coincidence with other studies regarding factors that traditionally affect the research training of students: curriculum, regulations and rules of the game, human resources, economic, material and technological resources, culture, leadership and individual abilities, however, innovation and interinstitutional linkage emerge as elements of tod ay's society.
\end{abstract}

Keywords:

Comparative education, Perception, Professors

\section{Resumen:}

El estudio analizó desde una perspectiva comparada, las percepciones de los profesores del área de Ciencias Sociales y Humanidades de dos universidades públicas: una mexicana y otra colombiana, sobre los factores que limitan la formación en investigación científica de estudiantes de la Licenciatura en Educación. Fue de tipo descriptivo y corte transversal. Se entrevistaron veintiún docentes: once profesores de la universidad mexicana y diez de la colombiana. Se compararon nueve factores con el modelo de Bereday. Los resultados revelan coincidencia con otros estudios en cuanto a factores que tradicionalmente inciden en la formación investigativa de los estudiantes: currículum, normatividad y reglas de juego, recursos humanos, recursos económicos, materiales y tecnológicos, cultura, liderazgo y capacidades individuales, sin embargo, la innovación y la vinculación interinstitucional emergen como elementos propios de la sociedad actual.

Palabras Clave:

Educación comparada, Percepciones, Profesores

a Universidad Juárez Autónoma de Tabasco, https://orcid.org/0000-0001-6692-0457, Email: jacinta.hernandez@ujat.mx

Fecha de recepción: 12/03/2020, Fecha de aceptación: 29/04/2020, Fecha de publicación: 05/06/2020 


\section{Introducción}

Históricamente el progreso social está aunado a la investigación, a la ciencia y a la tecnología. El desarrollo de estos factores son determinantes no solo para mejorar el presente, sino para crear más y mejores oportunidades en el futuro para los seres humanos. Es por ello que la formación de investigadores es factor clave en la política educativa de los países que pugnan por su desarrollo. Acorde a esto, la UNESCO emitió el documento "Recomendación sobre la Ciencia y los Investigadores Científicos" (2017), donde se establecen disposiciones dirigidas a las instituciones encargadas de la enseñanza científica, para favorecer la educación y la formación inicial de investigadores científicos: desarrollar técnicas didácticas que resalten la ética en la investigación, hábitos de pensamiento para el desarrollo del método científico, capacidad para analizar problemas con sentido humano y comunicación científica, entre otros.

En este sentido, la formación para la investigación en estudiantes de pregrado se torna sustancial para las universidades como instituciones de educación superior, pues no basta con formar estudiantes críticos, reflexivos, analíticos, conscientes y capaces de impactar en su entorno. Se requiere, como señala Edgar Morín, de estudiantes cuya formación investigativa les permita convertirse en egresados capaces de afrontar "realidades o problemas cada vez más pluridisciplinarios, transversales, multidimensionales, transnacionales, globales, planetarios" (1999:13). Y aunque la mayoría de los egresados de universidades "no harán investigación como su actividad principal, deben tener la capacidad de hacer investigación y realizarla cuando sea necesaria, en su campo laboral" (Miyahira, 2009).

La formación para la investigación en estudiantes de pregrado es un problema que no es privativo de un campo de conocimiento o una disciplina en particular. En la última década este tema se ha abordado en áreas como: Salud (Williams y Garcés, 2018; Abu-Zaid, \& Al-Kattan, 2013; Ruíz, Fernández y Horta, 2012), Derecho (Alarcón, 2014; Cabeda, 2010), Comercio (Zamora, 2014), Odontología (Espinoza, Cintra, Pérez y León, 2016) e Ingeniería (Viteri, Vázquez, 2016), entre otras.

Áreas en las que los autores coinciden en que la formación en investigación científica es determinada por diversos factores que están relacionados con el currículum, la infraestructura y el equipamiento de las instituciones, la cultura de las disciplinas y las capacidades de los profesores y estudiantes, principalmente. Algunas de las causalidades que se han analizado son: insuficiencias en la formación investigativa de estudiantes, principalmente de tipo teórico-metodológico (Álvarez y Orozco, 2011; Zamora, 2014); baja producción y baja calidad de trabajos de tesis en pregrado (Díaz et al., 2008); importancia de la integración investigación-educación a partir de la colaboración de estudiantes con estudiantes graduados y profesores en proyectos de investigación (Dooley, Mahon \& Oshiro, 2004); obstáculos que enfrentan las mujeres en carreras de ciencia o como científicas (Campbell \& Skoog, 2004); estudiantes con carencia de habilidades en la búsqueda de información (Casamayor, Figueroa y Herrera, 2009); carencia de aplicación de técnicas y métodos pedagógicos participativos en la formación investigativa (Rojas, 2009); insuficiencia curricular (Osada, Ramos y Ruiz, 2010); insuficiencia y deficiencia en planteamientos para el desarrollo de habilidades investigativas en planes de estudio, (Herrera, Fernández y Orta, 2012; Espinoza, Cintra, Pérez y León, 2016); envejecimiento de investigadores y falta de estudios de prospectiva (Perdomo y Valera, 2010); carencia de una política de jóvenes investigadores con énfasis en el pregrado (Perdomo y Valera, 2010, Torres, 2006); falta de respuesta por parte de instituciones educativas a las necesidades actuales en cuanto a formación para la investigación en los estudiantes (Quiñones y Vélez, 2004); falta de atención (o descuido) de universidades en este tipo de formación por su aprehensión a insertarse en las sociedades del conocimiento (Osada et al., 2010, Díaz, 2011); escasez tecnológica, infraestructura, falta de personas capacitadas y especializadas (Rojas, 2009, Díaz et al., 2008); tensiones entre mentores y estudiantes de pregrado al colaborar en proyectos de investigación (Dolan \& Johnson, 2010); entre otros.

Bajo este contexto es que se realizó esta investigación, cuyo objetivo fue determinar la situación que guardan algunos factores institucionales de dos universidades latinoamericanas, para formar en investigación científica a los estudiantes de la Licenciatura en Educación a partir de la percepción de los Profesores.

\section{Método}

\section{Contexto y Participantes}

Para el desarrollo del estudio se utilizó el método comparado con base en el modelo de Bereday (Bray, Adamson \& Mason, 2010), el cual señala que para analizar comparativamente se desarrollan primordialmente cuatro etapas: Descripción, interpretación, yuxtaposición y comparación (ver figura 1)

Figura 1. Modelo de Bereday del método de análisis comparativo.

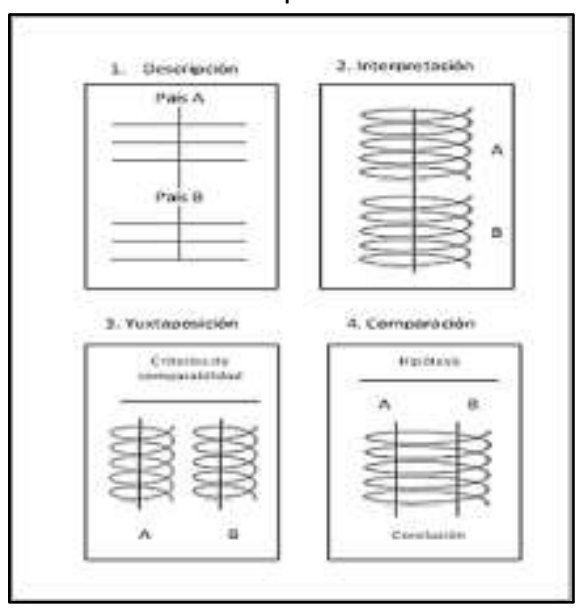

Fuente: Manzon (2010). 
La descripción se realizó en torno al contexto político, social, económico y cultural de los dos países, estados, localidades e instituciones participantes. La yuxtaposición se llevó a cabo a partir de una matriz de doble entrada en la que se confrontó la información obtenida, para posteriormente contar con elementos que permitieran la comparación.

México y Colombia son países que presentan grandes similitudes: ambos fueron conquistados y colonizados por españoles, antecedente que define sus rasgos culturales actuales, como el idioma y sus costumbres. Los dos se gobiernan por un régimen presidencialista. Su organización territorial también es semejante, México cuenta con 31 estados y un Distrito Federal; Colombia 32 Departamentos y un Distrito Capital. En el ámbito económico, el Banco Mundial registra un crecimiento porcentual similar del Producto Interno Bruto (PIB) en 2018, México: 2.1, Colombia: 2.6. El gasto en investigación y desarrollo (\% del PIB) que Colombia realizó en 2017 fue de 0,244, lo que representa una diferencia en cuanto a México, con 0,487 en 2016.

Los criterios de selección que determinaron a estos países como comparables fueron: a) Similitud en cuanto a indicadores de desarrollo social, económico y cultural, c) Factibilidad para la realización del estudio en términos de temporalidad y recursos económicos, d) Existencia de universidades públicas que ofertaran la Licenciatura en Educación o carrera afín y e) Que contaran con programas o políticas orientadas a la formación en investigación científica de los estudiantes. La selección de las universidades se basó en dos criterios: que ofertaran la licenciatura en Educación o carrera afín, y que contaran con políticas o programas orientados a la formación para la investigación científica en estudiantes.

En el caso de la universidad mexicana (UM), se imparte la Licenciatura en Ciencias de la Educación (LCE), cu ya matrícula era de 1271 estudiantes, con una planta docente de 101 profesores. En esta universidad se desarrollan los programas de Verano de la Investigación Científica y Jóvenes a la Investigación. Los estudiantes pueden participar como colaboradores en los proyectos de investigación de los profesores. La tesis es una de las 12 modalidades de titulación con las que cuenta este programa.

En la universidad colombiana (UC) se ofrece la Licenciatura en Ciencias Sociales (LCS), cuya matrícu la ascendía a 651 alumnos, con una planta docen te de 45 profesores. En esta universidad se desarrolla el programa de Semilleros de investigación. La tesis es la única modalidad de titulación para los estudiantes.

La Misión de la LCE incluye formar personas que hagan investigación y extensión relevantes para el desarrollo del país. La Misión de la LCS establece la formación de profesionales con capacidades y actitudes investigativas, críticas, reflexivas y propositivas en el campo de las ciencias sociales para el desarrollo humano. Teniendo como base el contexto antes descrito, en este trabajo se analizan comparativamente las percepciones de profesores del área de Ciencias Sociales y Humanidades que imparten clases en la LCE y LCS, por lo que en el estudio participaron un total de veintiún profesores.

\section{Técnica de investigación}

La recolección de datos se hizo mediante entrevista semiestructurada. La planta docente de la LCE de la UM estaba integrada por 101 profesores: 58 de tiempo completo, 7 de medio tiempo y 36 de asignatura, de los cuales: 16 tenían grado de Doctorado, 68 Maestría y 17 Licenciatura. La planta docente de la LCS de la UC estaba constituida por 45 profesores: 24 de tiempo completoii, 3 de medio tiempo, 3 de tiempo parcial y 15 de horas cátedra, de los cuales: 4 contaban con grado de Doctorado, 30 con Maestría, 9 con Especialidad y 2 con Pregrado.

Para seleccionar a los participantes se utilizaron dos estrategias de muestreo intencional: el muestreo homogéneo y bola de nieve (Creswell, 2012). En la UM se utilizó el muestreo intencional homogéneo, cuya principal característica es que quienes conforman la muestra pertenecen a un subgrupo que posee rasgos o características similares. La adscripción de la investigadora a esta universidad facilitó la identificación previa de profesores vinculados a la investigación, para posteriormente enfocarse a su localización. En la UC, fue necesario utilizar ambas estrategias "en algunos estudios puede ser necesario utilizar varias estrategias de muestreo diferentes (p.ej. seleccionar profesores en una escuela y seleccionar diferentes escuelas para ser incluidos en la muestra)" (Creswell, 2012:207). El muestreo homogéneo se utilizó para identificar en un primer momento a algunos profesores altamente relacionados con el área de investigación, con quienes se tuvo un primer acercamiento al entrar al trabajo de campo, convirtiéndose en informantes claves que a su vez orientaron para incorporar a otros profesores que reunían las características necesarias para el estudio. La muestra estuvo conformada por 21 profesores.

Los criterios de inclusión utilizados fueron: a) tuvieran experiencia en desarrollo de proyectos de investigación, b) impartieran o hubieran impartido asignaturas en esta área del conocimiento y/o c) hubieran desempeñado alguna función administrativa relacionada con el desarrollo de la investigación (ver tabla 1).

En la UM se entrevistaron 11 profesores: cinco hombres y seis mujeres, cuatro con grado de doctorado y siete con grado de maestría. Cinco de los profesores, además de su experiencia como docentes, se habían desempeñado en puesto administrativo, cuyas funciones estaban relacionadas con el fomento de la investigación científica en los estudiantes. En la UC se entrevistaron 
10 profesores: cuatro hombres y seis mujeres, uno con grado de doctorado y nueve con grado de maestría. Tres profesores habían desempeñado funciones administrativas vinculadas a la investigación.

Tabla 1. Profesores participantes

\begin{tabular}{|c|c|c|}
\hline \multicolumn{2}{|c|}{ Participantes } & \multirow{2}{*}{ Características conforme a criterios } \\
\hline UM & UC & \\
\hline 1,2 & 1,2 & $\begin{array}{l}\text { Experiencia en proyectos de } \\
\text { investigación }\end{array}$ \\
\hline 3,4 & 3,4 & \multirow[b]{2}{*}{$\begin{array}{l}\text { Experiencia docente en asignaturas } \\
\text { articuladas a la investigación }\end{array}$} \\
\hline 5,6 & $\begin{array}{ll}5, & 6, \\
7 & \\
\end{array}$ & \\
\hline $7-11$ & $8-10$ & Experiencia docente y administrativa \\
\hline \multicolumn{3}{|r|}{ Total: 21 Participantes } \\
\hline
\end{tabular}

Fuente: Elaboración propia con base en criterios de selección.

La guía de preguntas se diseñó a partir de nueve factores derivados de una indagación previa a través de entrevistas focalizadas a profesores y estudiantes, los cuales constituyeron los ejes de comparación (ver figu ra 2.).

Figura 2. Factores asociados a la formación en investigación científica en estudiantes de pregrado.

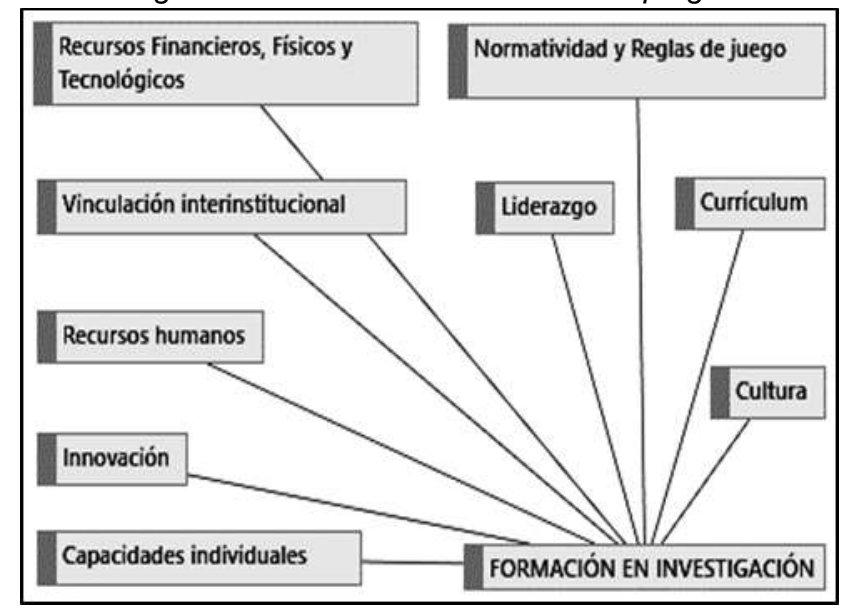

Fuente: Elaboración propia

Validez

La validez del instrumento se fundamentó en la revisión de tres investigadores expertos en el tema y en una prueba piloto, en la que se entrevistaron: dos profesores en la universidad mexicana y dos en la colombiana. Este proceso evidenció diferencias léxicas entre los dos países. Por esta razón, en Colombia se sustituyeron algunos términos por sus equivalentes: plan de estudios por pensum; director por decano; Maestro por Magister y División Académica por Facultad. Asimismo, se reemplazó el factor Relaciones interinstitucionales por Vinculación interinstitucional. Para validar los resultados se utilizó la estrategia de verificación de miembros (Creswell, 2012; Mertens, 2010) con entrevistas a tres profesores participantes, quienes apoyaron en la verificación e interpretación de la información.

Acceso a informantes y sitios

En ambas universidades se obtuvieron permisos en diferentes niveles para acceder a los informantes (Creswell, 2012). En México, por la Dirección de la División Académica y la Coordinación de Docencia. En Colombia, por la Oficina de Relaciones internacionales, Vicerrectoría de Investigación, Vicerrectoría de Docencia, Coordinación de Posgrado en Educación, Decanatura de la Facultad de Educación y la Coordinación de la carrera. La gestión en la universidad de Colombia se inició a distancia y se concretó dos días después de iniciar una estancia para la recolección de datos, que duró un mes. En ambos casos, se desarrolló una etapa de información y sensibilización para acercarse a los informantes clave, lo que incluyó entrevistas con directivos y coordinadores, para explicar los objetivos y la importancia de la investigación, presentar el instrumento de recolección de datos y garantizar el uso confidencial y académico de la información.

En las dos universidades se desarrolló la estrategia de establecimiento de relaciones con los responsables de las áreas señaladas (Flick, 2007), lo que permitió obtener información respecto al día, lugar y hora en que se podía contactar a los profesores, su perfil académico y profesional, así como algunas características de su personalidad. En el caso de Colombia, se añadió la estrategia de referencia, donde los coordinadores y profesores corrían la voz de la investigación que se estaba realizando, así como el objetivo de la misma.

\section{Desarrollo de entrevistas}

De acuerdo con las características de infraestructura, disponibilidad de tiempo y asignación de espacios a profesores, las entrevistas se realizaron tanto en ambientes cerrados como abiertos. En México prevalecieron los primeros: cubículos, biblioteca y aulas; mientras que en Colombia, fue en espacios abiertos: cafetería, explanadas y áreas de descanso ubicadas en la planta baja de los edificios, esto debido a que los profesores no contaban con cubículos y la mayoría tenía otras fuentes de empleo, por lo que solo imparten la asignatura y se retiran.

En México, los profesores mostraron una actitud colaborativa de manera inmediata. En Colombia, inicialmente se percibió una actitud de desconfianza y reserva respecto al uso que se le daría a los resultados de la investigación, lo que se dirimió con la garantía del anonimato, el carácter académico del estudio y el compromiso de regresar y presentar los resultados obtenidos, como retribución al apoyo brindado. Todas las entrevistas fueron grabadas en audio para su posterior análisis. Las notas de campo constituyeron una valiosa herramienta para el registro de observaciones e información relevante generada durante la interacción verbal. 
Análisis de datos

El análisis de datos se realizó con la técnica de análisis del discurso. Este tipo de análisis se "enfoca a la comprensión del lenguaje de los participantes" (Mertens, 2010:427). Se diseñó una matriz de análisis donde se realizó el proceso de: a) reducción de datos, b) extracción de significados y c) obtención de resultados. La reducción de datos se llevó a cabo mediante la separación de unidades de análisis.

El criterio que se utilizó en esta etapa fue el Gramatical (Rodríguez, Gil y García, 1996), a partir de la identificación de oraciones o párrafos con sentido lógico. La extracción de significados se desarrolló por medio de la categorización y codificación, lo que implicó un proceso de síntesis y agrupamiento de datos, para posteriormente obtener los resultados de la aplicación de la técnica. En este proceso se utilizó el programa de Excel y el Atlas Ti.

\section{Resultados}

El orden estructural en que se presentan los resultados corresponde al de los factores examinados, mismos que constituyeron los criterios de comparación. Cada uno contiene: a) las dimensiones analizadas, b) un ejemplo de pregunta, c) citas de los informantes para ilustrar con sus propias palabras algunos de los resultados, siendo la codificación: UM (Universidad Mexicana) / UC (Universidad Colombiana) $+E$ (Entrevista) + 0 (número de entrevista) y d) figura con las principales convergencias y divergencias identificadas de acuerdo a la percepción de los profesores de cada una de las universidades.

\section{1) Currículum.}

Las preguntas hicieron referencia a: Orientación del plan de estudios para el desarrollo de habilidades y competencias investigativas, cumplimiento de contenidos temáticos, desarrollo de actividades prácticas, asesoría y apoyo de profesores a estudiantes en actividades investigativas y articulación de asignaturas de investigación. Ejemplo: ¿A su juicio, qué competencias investigativas considera que desarrollan los estudiantes?

¿Qué desarrollen ellos? Hay algunos que las desarrollan: responsabilidad y sentido de ética. Pero si una competencia es el saber hacer y el saber estar, pues a veces no saben ni estar ni ser... deben fortalecer y tener habilidades cognitivas desarrolladas, competencias de análisis y síntesis y pues muchas veces no las desarrollan (UME2).

Totalmente se queda corto el desarrollo de análisis, crítica, comunicación. Esa es mi apreciación. Y le cuento que no son pocos, son bastantes, porque a mí me han tocado cursos con 40 o más, y al finalizar, y lo que yo veo, que quienes tienen el conocimiento son $10 \circ 12$, los demás no lo tienen (UCE5).
Los profesores de ambas universidades concuerdan en que los planes de estudios orientan a la formación investigativa de los estudiantes, sin embargo, estos presentan deficiencias en el desarrollo de sus competencias investigativas. Por otro lado, se evidencian problemas de articulación entre asignaturas, que en la UM se reflejan en la continuidad de contenidos temáticos: "...yo pensaría que, que antes de poder abordar, por ejemplo, eh... paradigmas, muestreo, mediaciones, yo creo que lo primero que deberíamos de hacer es tener una materia que sí habláramos del área general" (UME1); "No hay ninguna articulación, hay una fragmentación" (UME4).

De acuerdo con los profesores de UC, en el tiempo de formación que transcurre entre los primeros y últimos semestres

...la investigación formativa empieza en segundo y continúa hasta cuarto y ahí se interrumpe todo el proceso, no se vuelve hablar de investigación y el estudiante no tiene acercamiento a él hasta que llega al séptimo que toma la temática del trabajo de grado (UCE2).

En la UM se identifican contrastes en el desarrollo de actividades prácticas en torno a la investigación. En algunas ocasiones, estas carecen de apoyo por parte del profesor o denotan desvalorización en su proceso formativo, mientras que en otros sucede lo contrario: los estudiantes se sienten orientados y apoyados

Tengo las referencias de algunos chicos que definitivamente me dijeron: Bueno, es que a nosotros el profesor únicamente nos utilizó para mandarnos a las diferentes divisiones que aplicáramos cuestionarios" [y en otras, los estudiantes se sienten orientados y apoyados] "...él se sentía muy contento porque me dice: Bueno, es que, la Doctora me llevó a hacer el trabajo de campo, estuvimos en las entrevistas, estuve grabando, me va a enseñar a usar el Atlas y vamos a hacer la interpretación; a partir de esto, puedo ver que hay un proceso guiado, hay un acompañamiento" (UME6) (ver figura 3).

También se manifestó la omisión de contenidos temáticos: "y además, una queja muy clara inclusive, porque eran alumnos, que ni les dio las clases de la materia que debería de darles" (UME5). En contraste, los docentes de la UC declararon que generalmente se cubren los contenidos temáticos, sin embargo, los estudiantes no desarrollan actividades investigativas prácticas, solo su trabajo de grado (tesis), principalmente porque los profesores no investigan y es la única opción de titulación. 
Figura 3. Convergencias y Divergencias: Currículum.

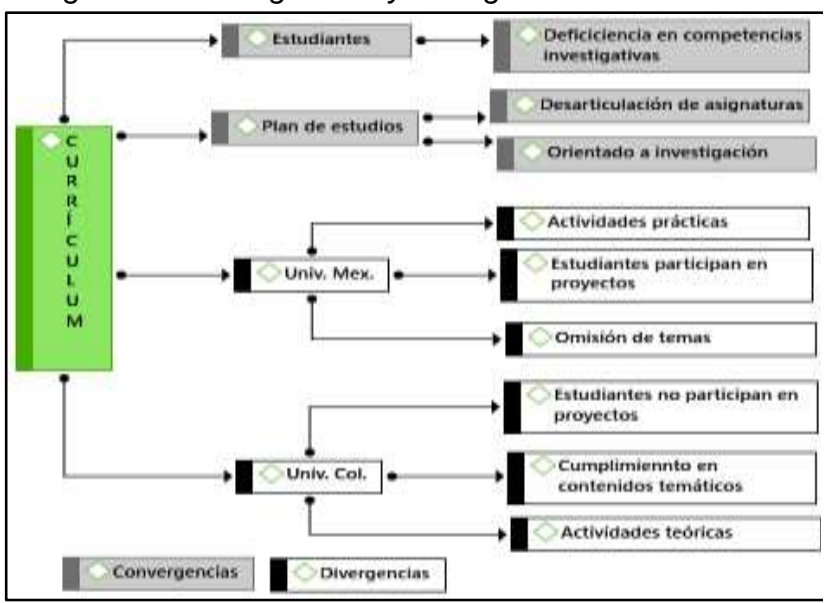

Fuente: Elaboración propia

\section{Normatividad y Reglas de juego.}

Esta categoría comprendió preguntas relacionadas con: Importancia de la investigación en las políticas institucionales, difusión de programas orientados a la investigación en los estudiantes, conocimiento de reglas de juego por estudiantes, asignación de materias a profesores, diversificación de medios para difundir programas para fomentar la investigación en los estudiantes, asignación de materias a profesores que desempeñan otros cargos. Ejemplo: ¿De qué manera se difunden los programas de investigación en los estudiantes?

Se difunden las convocatorias a través de circuito cerrado, por oficio a presidentes de academia y bajan a los profesores, carteles... también la visita a los salones ha sido una parte muy importante, para atender y aclarar dudas, hasta se ha invitado a otro par de ellos [estudiantes], que ya fueron y platiquen sus experiencias (UME6).

Una divulgación como tal, de los semilleros, de los referentes de investigación, muy poca hay, como que esa es una falencia... no hay medios de comunicación para difundir entre los estudiantes, si hacen una convocatoria de semilleros ponen una panfletita a casi 25000 metros, entonces ¿Quién los va a ver? (UCE2).

Los docentes de ambas universidades coinciden en que las políticas y fundamentos institucionales consideran la investigación como un factor trascendental en la formación de los estudiantes, sin embargo "aunque la misión de la universidad, la misión de la facultad y la misión del programa hablan de formación en investigación, se presentan situaciones que no corresponden a esa intencionalidad" (UCE1). En cuanto a la difusión se identifican diferencias entre las universidades, en la UM se utilizan diversos medios, y en la UC se señaló que estos son exiguos, lo que genera desconocimiento por parte de los estudiantes (ver figu ra 4).

Respecto a la asignación de materias, los entrevistados evidenciaron prácticas que no consideran la evaluación o experiencia en investigación de los profesores: "La coordinadora pasa un listado de las materias que se requieren y vicerrectoría asigna los profesores, vice docencia digamos que hace una función de que tenga las horas, que el profe pueda... (UC6).

Figura 4. Convergencias y Divergencias: Normatividad y Reglas de juego.

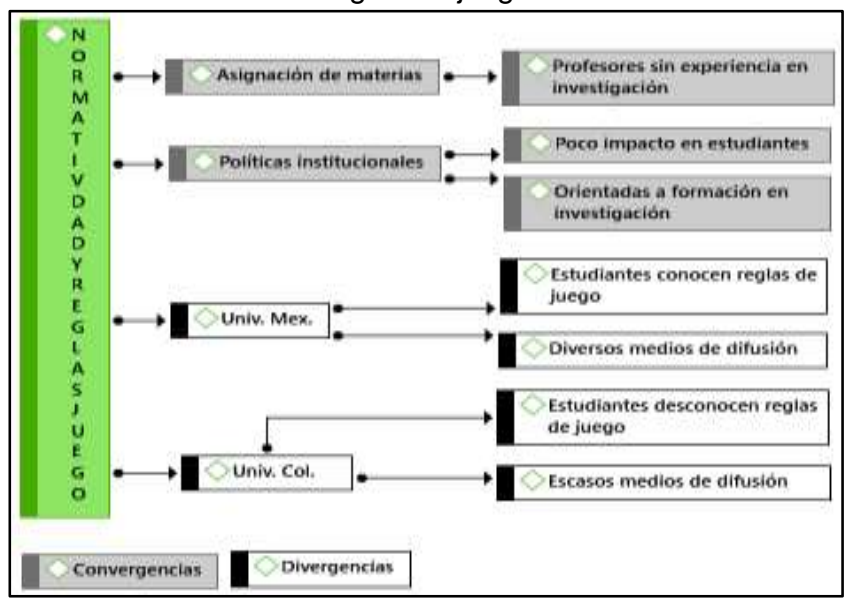

Fuente: Elaboración propia

\section{Recursos financieros, físicos y tecnológicos.}

Incluyó cuestionamientos sobre: Suficiencia de equipo de cómputo para estudiantes, situación de espacios físicos (aulas, salas, etc.), servicio de internet y apoyo económico a estudiantes para actividades de investigación. Ejemplo: ¿Qué opina del servicio de conexión a internet a estudiantes?

No hay servicio gratuito para conexión de estudiantes, maestra... no hay (UME4).

La plataforma no es suficiente para toda la comunidad estudiantil y profesores, y resulta muy deficiente, se vuelve lento (UCE2).

En cuanto a las aulas, los profesores de la UM señalaron la falta de aires acondicionados como un problema que afecta la práctica edu cativa "el calor es insoportable en el salón, en algunas áreas se ha puesto clima, pero los propios alumnos los descomponen" (UME8). En la UC se manifestó que la infraestructura física está en buenas condiciones, sin embargo, señalaron insuficiencia de mobiliario escolar y carencia de equipo tecnológico en aulas y en salas de cómputo "si yo quiero dotación la tengo que alquilar, alquilo el video proyector, el televisor, la grabadora. $Y$ algunas aulas dotadas si existen pero los estudiantes no pueden tener acceso porque son para algunas facultades, no para los de educación" (UCE3). 
Respecto a recursos económicos, en la UM los entrevistados manifestaron que los estudiantes sí reciben apoyo para realizar actividades de investigación "algunos de los apoyos que reciben son las becas tesis, becas para que hagan una estancia de Verano de la Investigación científica, o las que a veces les dan algunos profesores a quienes colaboran en alguno de sus proyectos" (UME6), situación que difiere de la UC, donde se manifestó que los estudiantes carecen de apoyos económicos en actividades relacionadas con la investigación, las cuales, además de ser insuficientes, cuando se realizan son solventadas por los propios estudiantes o profesores (ver figura 5).

Figura 5. Convergencias y Divergencias: Recursos financieros, físicos y tecnológicos.

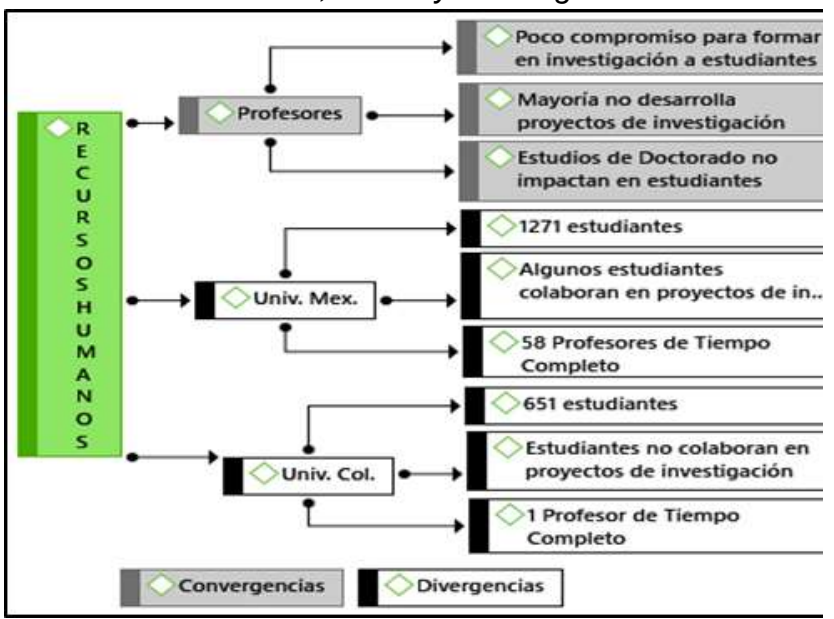

Fuente: Elaboración propia

De acuerdo a los entrevistados, esta situación obedece principalmente a:

a) Desigualdad en otorgamiento de apoyos, favoreciendo a programas con mayor trayectoria investigativa:

Yo diría que... que a nivel de la facultad nuestra, es poco lo que la uni (sic) invierte en ese sentido, pero sé que en otras como Economía, Ingeniería, Arquitectura, hace contribuciones la administración para el cuerpo docente y para estudiantes, especialmente en participación en eventos tanto nacional o internacional" (UCE8).

b) Prácticas de clientelismo político "Allí muchas veces prevalece el amiguismo, las influencias, las relaciones, que se tengan con los administradores en turno" (UCE5). "todo depende, si tiene amigos en ciertos sectores, tiene mucha más oportunidad" (UCE8); "el apoyo de investigación es nula; la vicerrectoría tiene todo un discurso, pero en la realidad, si hay apoyos, pero muy escasos tanto en cantidad como en número de apoyos y no son transparentes" (UCE3).

\section{Recursos humanos.}

Se hizo referencia a: Participación de profesores y estudiantes en proyectos de investigación, profesores con doctorado y su beneficio en la formación investigativa de los estudiantes en relación a los que tienen grado de maestría. Ejemplo: ¿Qué opinan de los profesores con grado de Doctor con respecto a su responsabilidad y compromiso en la impartición de asignaturas?

Tengo referencias negativas y positivas, hay gente que tiene grados topes, de doctorado, pero sus conductas no son adecuadas, sus comportamientos son de sentirse vacas sagradas, íconos inmovibles... cumplen a veces por cumplir, pero no con ese sentido de responsabilidad, pero también hay algunos que se preocupan porque los estudiantes hagan investigación (UME1).

Que los profesores que están en investigación, que son doctores, se note su trabajo en los programas, que no estén solo por ganar dinero, sino que se vea la proyección. $Y$ es verdad que muchos hacen doctorado pero no aplican lo que trajeron (COE9).

En la UM los entrevistados manifestaron que la mayoría de los profesores no hacen investigación "Los profesores tienen el grado, tienen todo, pero les falta formación de investigadores (...), la mayoría no lo hace (UME4). En la UC los docentes coinciden en que solo algunos profesores que estudian doctorado hacen investigación, y que al no haber semilleros de investigación en el programa, los estudiantes no tienen la oportunidad de colaborar en proyectos (ver figura 6) "no tenemos semilleros, no hay semilleros en el programa, y aunque quieran los muchachos no pueden integrarse, la única investigación que hacen es su trabajo de grado" (UCE1).

Cabe mencionar que en la UC resaltaron dos aspectos que de acuerdo a los profesores, limitan la formación investigativa de los estudiantes, uno es la falta de profesores de tiempo completo (PTC) "No hay PTC porque los docentes son de la uni y no de la facultad. La uni tiene un número de PTC que como colegio pudiera servir, pero como uni no sirve" (UCE2), y el otro, la politic:

Y otra cuestión es la política. Esta uni está en manos de políticos de la región y a un político le interesa más tener cuarenta docentes por hora que cuarenta PTC, porque los puede cambiar...y los PTC no los puede mover" (UCE2).

La uni está mediada por la politiquería... pero una cosa es hacer la politiquería con altura, ¿en qué sentido?, si bien colocaste a personajes que políticamente ayudaron al senador, hay que mirar qué tipo de gente se coloca, que sepa, que sepa que es lo académico (UCE1; UCE7). 
Figura 6. Convergencias y Divergencias: Recursos humanos

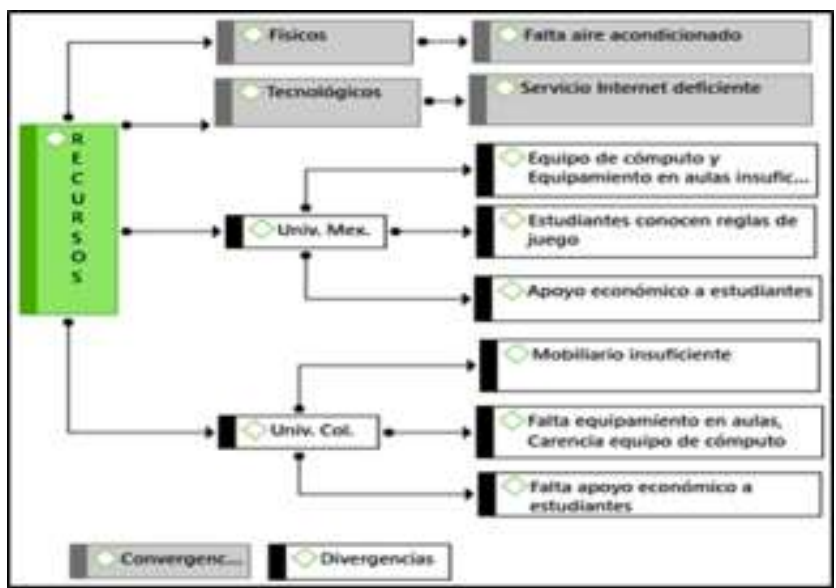

Fuente: Elaboración propia.

\section{Cultura.}

Implicó aspectos vinculados con: Interés de estudiantes por la investigación, realización de eventos que fomentan la investigación en estudiantes, participación de profesores y estudiantes en eventos y cultura investigativa de la Licenciatura. Ejemplo: ¿Cómo describiría usted las actitudes y creencias de los estudiantes con respecto a la investigación?

El alumno busca esa complicidad con el profesor que no lee... buscan a profesores que no dejen trabajo, no dejen leer. A veces se dan de baja y buscan otro maestro que no les exija (...) tienen miedo de hacer tesis, porque se les hace algo muy difícil, los mismos maestros se los dicen (UME3).

Los estudiantes muestran desinterés en la investigación, pero es porque no se les motiva. No hay semilleros en la carrera, por eso no participan en proyectos, pero saben que tienen que hacer una tesis para titularse (COE10).

Respecto a la realización de eventos que fomentan la investigación y la participación de profesores y estudiantes, en la UM se manifestó que "si hay algu nos eventos en que algunos estudiantes participan, au nque aún son pocos, como la semana de difusión científica" (UME5). En la UC la falta de infraestructura y apoyos económicos, obstaculizan la realización de este tipo de actividades "Muy complicado, muy complicado, nosotros tenemos estudiantes a la palestra... falta de recursos, no nos prestaron los salones adecuados (...) si tenemos que hacer la propaganda lo tenemos que hacer los estudiantes y los profesores, eso lo costeamos nosotros" (UCE8) (ver figura 7).

En ambas universidades se percibe la necesidad de fortalecer la cultura investigativa a través de diversas actividades "hace falta ir fomentando la cultura de la investigación en los jóvenes a través de talleres, a través de cursos, a través de una serie de estrategias, con el objetivo de querer formar una cultura investigativa en los alumnos (UME1).

En la UC los profesores puntualizaron que el contexto político, social y económico de la región determina las características cognitivas de los estudiantes, lo que influye en la escasa cultura investigativa en el programa educativo "Ios estudiantes nuestros vienen de los estratos más bajos del departamento, de la región y de la ciudad, por lo tanto traen una cantidad de falencias: cognitivas, afectivas, alimentarias, porque proceden de instituciones de baja calidad, de lugares marginados (UCE3).

Figura 7. Convergencias y Divergencias: Cultura.

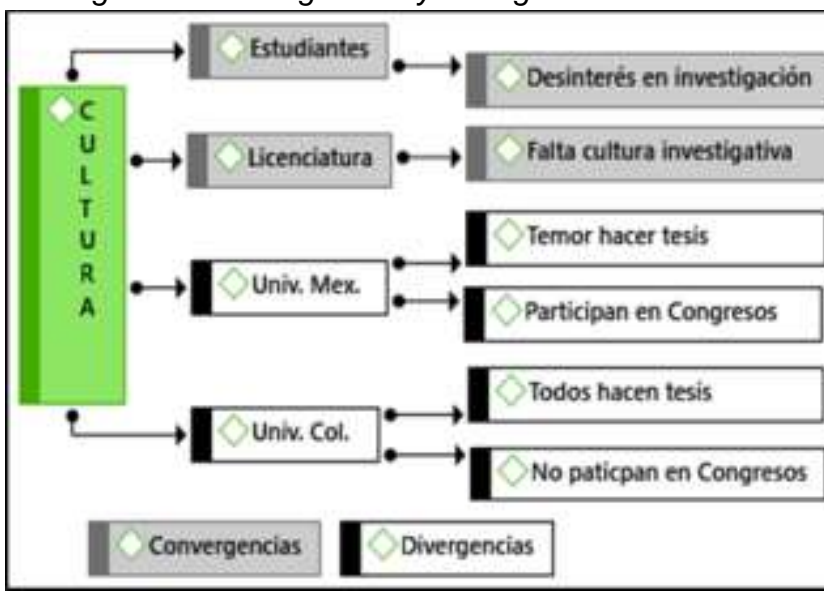

Fuente: Elaboración propia.

\section{Liderazgo.}

Incluyó preguntas relacionadas con: la motivación que ejercen los estudiantes con experiencia en investigación, profesores y personal responsable de programas, en los estudiantes para formarse en investigación. Ejemplo: ¿Qué formas de motivación ejercen los profesores para fomentar la investigación en los estudiantes?

No, es que luego nos desmotivan, sí, me lo han dicho concretamente, hay casos en los que los profesores dicen: No, no, no, no hagan eso [tesis] porque va a ser muchas vueltas, porque tiene que ir por los sinodales, porque se los van a regresar, porque les van a desbaratar los trabajos de tesis (UME5).

Lo que pasa es que como casi no se hace investigación aquí... lo único que ellos hacen es su trabajo de grado, y es obligatorio, si se quieren titular... entonces pues como no hay semilleros de investigación pues no se les puede motivar... (COE9).

En la UM se manifestó que los estudiantes con experiencia en investigación son agentes motivadores para que sus compañeros se formen en esta área, ya que ellos han participado en programas de Verano "Los estudiantes que han ido a Verano, son pieza importante para motivar a sus compañeros y nos están apoyando 
con eso, se organiza un foro donde trasmiten su experiencia. También se les motiva dándoles una beca para la impresión de su tesis" (UME6).

En el caso de la UC no se da esta dinámica, ya que los estudiantes no desarrollan investigación "Sí han participado estudiantes en estancias de investigación a nivel nacional, pero no de la Facultad de Educación. No se motivan tampoco porque la facultad no se ha preocupado por eso" (UCE3) (ver figura 8).

Figura 8. Convergencias y Divergencias: Liderazgo

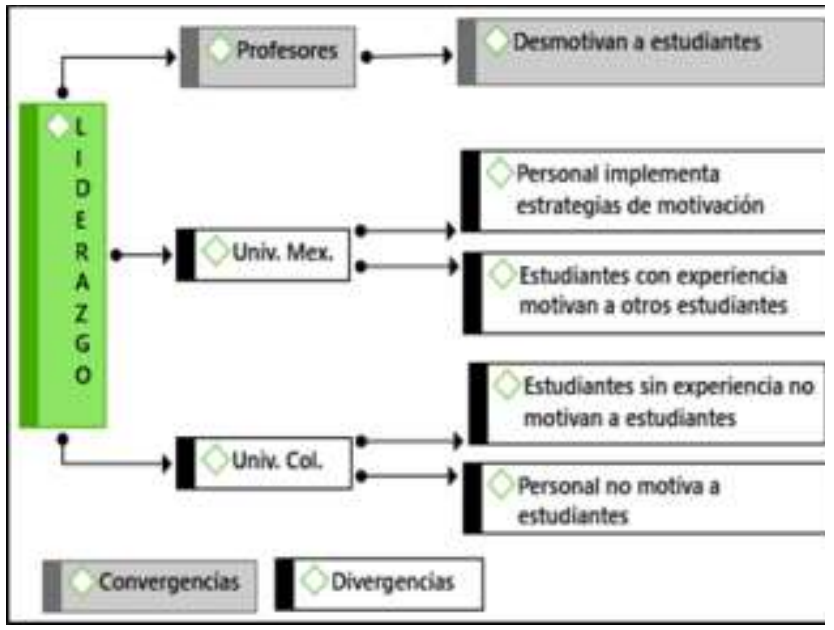

Fuente: Elaboración propia.

\section{Vinculación interinstitucional.}

Comprendió preguntas relacionadas con la investigación que se realiza con otras organizaciones a nivel local, nacional o internacional. Ejemplo: ¿Qué sabe usted sobre la investigación que se desarrolla a nivel local, nacional o internacional?

En materia de investigación ahí estamos en la parte endeble, creo que es necesario, indispensable, signar convenios con instituciones, con otras instituciones nacionales, internacionales, que apoye esta parte de la investigación con los estudiantes (...), y bueno, la investigación que hacen los profesores es más local que nacional o internacional... creo que uno de los talones de Aquiles (UME7).

Creo que deberíamos empezar por hacer investigación en lo regional. Se tiene vinculación con instituciones educativas para que los estudiantes vayan, practiquen... pero no de investigación. Ha habido intentos, pero como no hay apoyo, eso es una falencia aquí (COE8).

En ambas universidades los profesores manifestaron que cuentan con convenios tanto nacionales como internacionales, sin embargo, estos no contribuyen a la formación investigativa de los estudiantes

En la UC se enfatizó que existe poca vinculación con organismos del entorno local y regional, aludiendo que en la mayoría de los casos ésta favorece las prácticas pedagógicas de los estudiantes, dejando de lado trabajos de investigación "convenios aquí hay de los que tú quieras, de papel... pero no son reales. No tenemos acercamiento a la comunidad, a la industria, al comercio. Están los convenios escritos, pero no son realizados, ni con las universidades, ellas son ellas y nosotros somos nosotros, somos unas islas" (COE3) (ver figura 9).

Figura 9. Convergencias y Divergencias: Vinculación

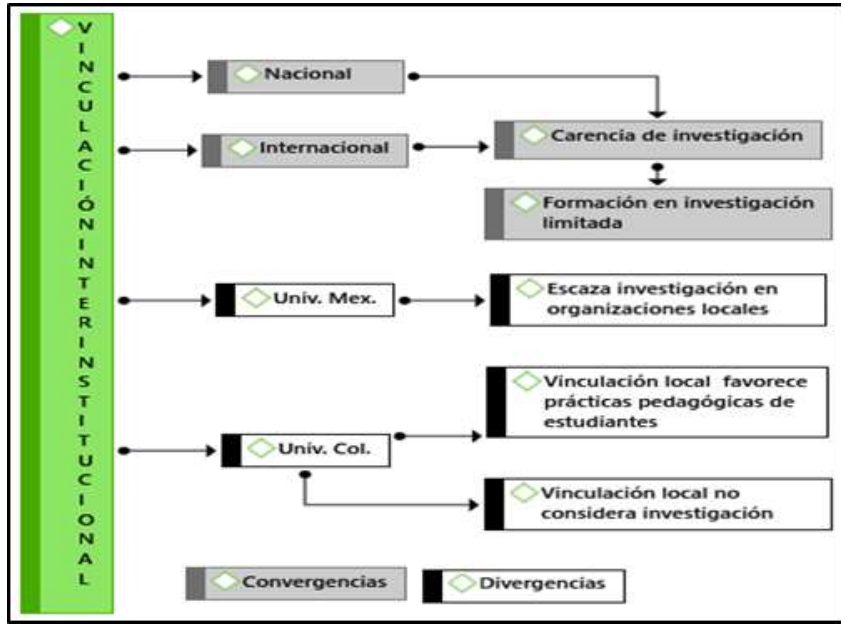

Fuente: Elaboración propia.

\section{Capacidades individuales}

En este rubro se plantearon aspectos vinculados con las capacidades individuales de los actores en cuanto a: habilidades tecnológicas y comunicativas, capacidad para comunicarse en otro idioma. Ejemplo: ¿Qué piensa sobre las habilidades tecnológicas que poseen los estudiantes para desarrollar actividades de investigación?

Los jóvenes requieren esa habilidad, porque ya están saliendo otros programas y ellos no tienen esa facilidad. Hay jóvenes que sí hacen uso de tecnología, como Excel, Power Point y otros, pero en cuanto a programas para investigación, les hace falta (UME6).

Muy limitadas, ellos utilizan esas habilidades para juegos, para subir mensajes, pero no se reflejan, al contrario, parecen un obstáculo, porque por estar jugando se distraen, lo podrían emplear para investigar... bajan chistes, juegos, etcétera. pero no es una herramienta de trabajo (COE3).

Los entrevistados refirieron que la comunicación de los docentes con los estudiantes presenta barreras de tipo semántico, lo que propicia desinterés o rechazo por la investigación "entonces el profesor cuando llega con un lenguaje que va superado por mucho a lo que man eja el alumno por todos los vacíos que traemos formativos, entonces el alumno, en vez de querer, aprende a rechazar" (UME1). Asimismo, se manifestó que existe escasa interacción entre profesores y estudiantes en el proceso de formación "Hay poca comunicación con los 
alumnos, porque uno entra, maneja el discurso con los estudiantes, depende de uno si los pone a leer, genera discusión, pero lo importante es llegar, cumplir, tirar el rollo y hacer evaluaciones" (UCE7) (ver figura 10).

Figura 10. Convergencias y Divergencias: Capacidades individuales

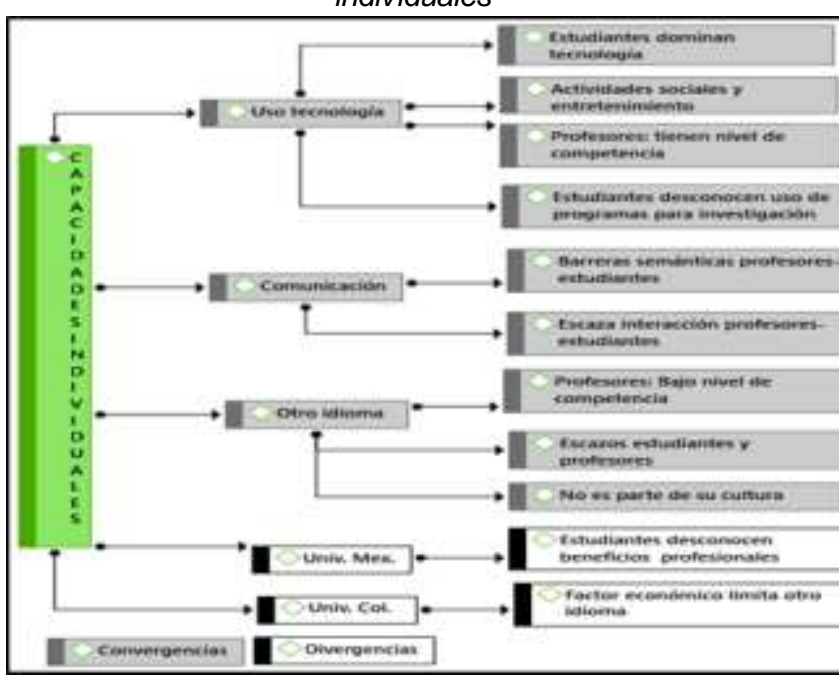

Fuente: Elaboración propia.

Al cuestionar sobre la capacidad de los alumnos para comunicarse en un segundo idioma, se identificó que existe un bajo nivel de dominio: "La gente aquí no tiene dominio de inglés. Es un requisito obligatorio que la gente para poderse graduar tiene que presentar un examen de esa segunda lengua, pero lo hace para graduarse, pero no forma parte de la cultura (UCE10), lo que a su vez se convierte en una limitante para el desarrollo de estancias de investigación en ámbitos internacionales.

"Agraciada o desgraciadamente, ahorita las convocatorias que están abiertas para hacer estancias internacionales son en países de habla inglesa, entonces las oportunidades para los estudiantes de educación se corta un poco, porque eso es una limitante, el idioma" (UME6).

\section{Innovación}

Incluyó cuestionamientos sobre trabajo colaborativo de profesores y estudiantes, centros 0 áreas de investigación y diversificación de fuentes de financiamiento. Ejemplo: ¿Qué opina usted sobre los centros 0 áreas de investigación destinadas a actividades de investigación?

Pues tenemos el Centro de investigación, pero es un espacio muy pequeño, para solo unos profesores y pocos estudiantes, eso es insuficiente... espacios especiales para investigar no tenemos, bueno, creo que faltan recursos económicos, que a lo mejor la universidad no tiene (UME9).
No hay, aquí los grupos académicos se disolvieron de miedo a una revuelta, de miedo a una insurrección, y tú ves que no existen salas de estudio, sala de profesores, lugares para los profesores donde puedan atender a los estudiantes (UCE3).

Los entrevistados desvelaron la falta de espacios para hacer investigación, sin embargo, en la UC se hizo referencia y además se constató, que los estudiantes integrados a Semilleros de investigación sí cuentan con una sala exclusiva para su uso, en donde no confluyen los estudiantes de Educación "desgraciadamente, los estudiantes de la Licenciatura en Educación no pueden ingresar a estos espacios porque no pertenecen a ningún Semillero, porque no hay ningún Semillero registrado en esta carrera" (UCE10).

Desde la percepción de los profesores de la UC, el trabajo colaborativo está ausente, esto debido a varios factores: escasa comunicación, actitudes regionalistas, falta de profesores de tiempo completo y politics: "entre los docentes no existen canales de comunicación, empezando porque el programa no tiene profesores de planta" (UCE4).

Entonces la política es que entre más lejos esté cada uno de cada uno. No hay una comunidad académica porque no hay donde reunirse ni hay espacio, ni tiempo. Entonces no hay ni política, ni espacio, ni voluntad, porque se cree que dos personas están confabulando y pueden generar situaciones no convenientes (UCE3).

Los entrevistados de la UM manifestaron que se cu enta con diversas fuentes de financiamiento para apoyar a los estudiantes en actividades de investigación "Para estancias de verano, se les apoya con becas institucionales, otras vienen del Consejo de Ciencia y Tecnología del estado de Tabasco, también se les apoya para que asistan como ponentes a congresos" (UME6).

En contraste, los profesores de la UC señalaron que el apoyo de la universidad tanto a estudiantes como a docentes para desarrollar actividades investigativas es casi inexistente, y que las actividades que realizan son autofinanciables (ver figura 11):

No, aquí eso es individual, aquí cada profesor, en el caso individual, yo me pago, yo lo hago por aparte" (UCE5)

...hacemos acercamientos pero son individuales, personales. Soy de Antioquia, voy y cuando llego digo que fui a Antioquia, entonces lo ponen como si fuera de aquí. Luego lo vuelven institucional. Pero fui con mi plata" (UCE6). 
Figura 11. Convergencias y Divergencias: Innovación

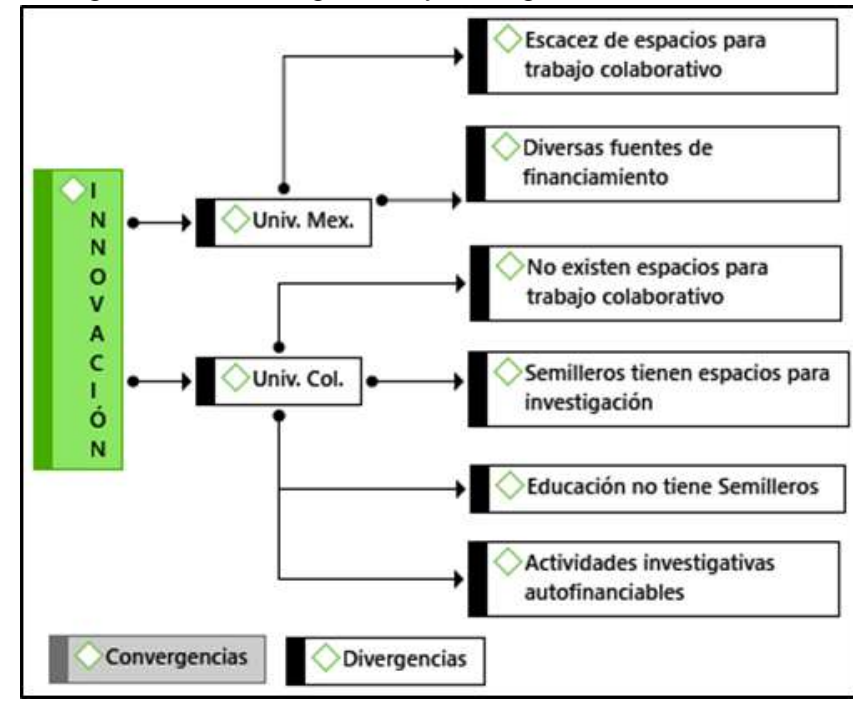

Fuente: Elaboración propia.

\section{Discusión}

El objetivo principal fue analizar comparativamente la percepción de los profesores de la licenciatura en Educación de dos universidades públicas: una mexicana y otra colombiana, respecto a nueve factores que inciden en la formación investigativa de los estudiantes, los cuales son: Currículum, Normatividad y reglas de juego, Recursos financieros, físicos y tecnológicos, Recursos humanos, Cultura, Liderazgo, Vinculación interinstitucional, Capacidades individuales e Innovación, cada uno con diversos componentes. El estudio permitió identificar las convergencias y divergencias entre las percepciones del profesorado en ambas instituciones para formar en investigación a los estudiantes y se visualiza que presentan algunas de las más relevantes semejanzas con estudios realizados por otros autores.

De acuerdo con la percepción de los profesores, las convergencias están correlacionadas con el escaso desarrollo de competencias investigativas en estudiantes de nuevo ingreso, que devienen de su formación en educación media superior (Rojas \& Méndez, 2017). En la práctica docente, los profesores desarrollan parcialmente los contenidos temáticos, acompañados de una didáctica tradicional, lo que está estrechamente vinculado con el escaso desarrollo de habilidades tecnológicas de los profesores, en contraposición de los estudiantes, quienes las aplican primordialmente en redes sociales; poca motivación de profesores a estudiantes, pese a que se reconocen a sí mismos como actores determinantes en la formación investigativa de los estudiantes y en la actitud de estos hacia actividades investigativas (Betancourt, 2017).

Otras variables que se perciben como limitantes en la formación en investigación de los estudiantes son: insuficiencia en equipo de cómputo y deficiencias en el servicio de internet a los estudiantes; baja calidad del servicio que brinda el personal administrativo responsable de fomentar la investigación en los estudiantes, que valga mencionar, en ambos casos dicho personal se mostró desmotivado en el desarrollo de sus actividades, pese a que se identificó que en la universidad mexicana sí reciben un salario por su labor, no así en la universidad colombiana. Asimismo, se detectó poco compromiso de doctores para formar en investigación científica a los estudiantes, por lo que se percibe que la maestría y doctorado no son niveles determinantes en la formación investigativa. Existe escasa cultura investigativa en ambos programas (Rojas, 2008), además, de que dos de los hallazgos que sobresalen en relación con otros estudios, son la vinculación interinstitucional e la innovación.

Algunas limitaciones del estudio son que los resultados se circunscriben a dos universidades, en las que prevalecen las similitudes en el ámbito institucional, geográfico, social y económico, por lo que quizá realizar estudios comparativos en otros contextos en los que prevalezca la diferencia en estos aspectos, resultaría de gran valía para identificar buenas prácticas que favorezcan la formación investigativa de los estudiantes. Desde el punto de visto metodológico, una limitación que se afrontó fue la escasez de estudios de educación comparada que describieran el método, lo que se visualiza como una necesidad en el desarrollo de este tipo de estudios, ya que brindaría pautas que facilitarían el trabajo e innovación de otros autores interesados en el análisis comparativo. No obstante las limitaciones, los hallazgos permitieron retroalimentar a docentes $y$ directivos de las universidades participantes, posibilitando el diseño de estrategias orientadas a fortalecer la formación en investigación de los estudiantes.

\section{Conclusiones}

De los resultados obtenidos, se pueden resaltar algunas conclusiones respecto a los factores que limitan la formación investigativa de los estudiantes de la licenciatura en Educación de las universidades participantes, las cuales se ciñen al contexto donde se realizó el estudio. El estudio demostró, en coincidencia con otras investigaciones, que el reto de las universidades para formar en investigación científica es multifactorial. Sin embargo, pese a coincidir en aspectos relacionados con el currículum, la normatividad, los recursos, la infraestructura y la cultura, sobresalen dos que cobran mayor relevancia en el contexto actual, el de Vinculación interinstitucional y el de la Innovación.

Respecto a la Vinculación interinstitucional, el estudio ha mostrado que pese a la existencia de convenios entre las universidades y organizaciones de diferentes ámbitos, el 
desarrollo de proyectos de investigación entre ambas partes es exiguo. Esto pone en relieve la necesidad realizar estudios que profundicen sobre los vínculos que realizan las universidades con otras instituciones, además de las acciones que a partir de estas se desarrollan para fortalecer la formación en investigación, no solo de sus profesores, sino también de sus estudiantes, es decir, ¿Cuántos y con qué tipo de convenios cuentan? ¿Qué impacto han tenido los convenios existentes en la formación investigativa de los alumnos? ¿Cómo se relacionan las universidades con otros sectores sociales para realizar investigaciones en las que participen los estudiantes? Serían algunas de las indagatorias que abriían nuevas vetas en este campo temático. Esto cobra relevancia si se considera que generalmente las universidades públicas tienen convenios signados con otras instituciones de educación superior, tanto nacionales como extranjeras, lo que además constituye en la mayoría de los casos, indicadores para procesos de evaluación institucional.

En cuanto al factor de Innovación, los profesores perciben que las principales dificultades para formar en investigación a los estudiantes están relacionadas con: la poca disponibilidad de espacios físicos, situación que prevalece en el quehacer de los docentes y que se hace extensivo a los estudiantes; el arraigo de una visión unidisciplinaria en trabajos de investigación y la falta de trabajo colaborativo e interdisciplinar, que si bien esta última se demanda de los profesores, habría que considerar en futuras investigaciones qué se está haciendo con los estudiantes en esta materia ¿Cómo se incorpora en el proceso de enseñanza y aprendizaje? ¿Qué actividades se desarrollan dentro y fuera del aula para fomentar la interdisciplinariedad 0 transdisciplinariedad en los estudiantes de licenciatura? Formar en investigación científica a estudiantes de la Licenciatura en Educación, es un gran reto que enfrentan las universidades ante una sociedad, que si bien es cierto ha evolucionado exponencialmente en materia de tecnologías de información y comunicación, también lo es que aún afronta problemas que demandan profesionales que contribuyan a su solución, ya sea desde la cotidianeidad de la vida, de su trabajo en el campo laboral, de su desempeño como científicos sociales o simplemente como formadores de formadores.

\section{Referencias}

Abu-Zaid, Ahmed \& Al-Kattan, Khaled (2013) "Integration of scientific research training into undergraduate medical education: a reminder call" en Medical Education Online No. 1, October. Bethesda: U.S. National Institutes of Health's National Library of Medicine. Disponible

https://www.ncbi.nlm.nih.gov/pmc/articles/PMC3805839/ [8 de febrero de 2019].
Alarcón Lora, Andrés Antonio (2014) "La investigación en la enseñanza del derecho para la formación de abogados. Caso Universidad de Cartagena de indias Periodo 1994 - 2014" en Saber, ciencia y libertad [En línea] No. 2, Julio. Cartagena: Universidad Libre. Disponible en: https://revista s.unilibre.edu.co/index.php/saber/article/view/2068/1506 [21 de marzo de 2019].

Álvarez Villar, Víctor Manuel \& Orozco Echavarría, Oilda (2011). "Determinación de la formación investigativa en estudiantes de carreras de ciencias sociales y humanísticas de la sede universitaria municipal de Guantánamo", en Cuadernos de Educación y Desarrollo [En línea] No. 25, Enero. Málaga: Universidad de Málaga. Disponible en: http://www.eumed.net/rev/ced/25/avoh.htm [22 de marzo de 2018]

Campbell, Ashley \& Geral, Skoog (2004) "Preparing undergraduate women for science careers Facilitating Success in Professional Research" en Journal of College Science Teaching [En línea] No. 5, Marzo. New York: College Science Teaching. Disponible en: https://www.nsta.org/publications/news/story.. spx ?id=49129 [15 de marzo de 2019].

Casamayor LaimeI, Zuleika, Figueroa LescailleII, Mercedes \& Herrera Pedroso, Amaira (2009) "La formación científico investigativa en los cadetes de la carrera de Medicina como problema pedagógico" en Revista Cubana de Medicina Militar [En Línea] No. 2, abril. La Habana: Universidad de Ciencias Médicas de las FAR. Disponible en línea en: https://pesquisa.bvsalud.org/portal/resource/pt/lil-547107 [20 de marzo de 2019].

Caveda, Alfonso (2010) "La formación investigativa en la carrera de derecho: los estudios jurídicos en la Universidad de Pinar del Río" en Odiseo. Revista electrónica en pedagogía [En línea] No. 15, Diciembre. Querétaro. Disponible en: https://www.odiseo.com.mx/2010/8-15/pdf/caveda-estudiosjuridicos.pdf [20 de marzo de 2019].

Creswell, John. (2012). "EducationalResearch: Planning, Conducting, and Evaluating Quantitative and Qua litative Research" [En línea]. Boston: Pearson. Disponible en: http://basu.nahad.ir/uploads/creswell.pdf [28 septiembre 2019].

Díaz Vélez, Cristian, Manrique González, Luis Miguel, Galán Rodas, Edén \& Apolaya Segura, Moisés (2011) "Conocimientos, actitudes y prácticas en investigación de los estudiantes de pregrado de facultades de medicina del Perú" en Acta Médica Peruana [En línea] No. 25, Enero. Lima: Colegio Médico del Perú. Disponible en línea en: http://www.redalyc.org/articulo.oa?id=96625103 [22 de marzo de 2018].

Díaz, Víctor Patricio (2011) "Relación entre sociedad del conocimiento, metodología de la investigación científica y producción científica estudiantil en estudiantes de medicina" en Revista Colombiana de Medicina [En línea] No. 3, Julio. Chile: Universidad del Valle. Disponible en línea en: Recuperado de www.scielo.org.co/pdf/cm/v42n3/v42n3a17.pdf [30 de agosto de 2018].

Dolan Erin, Johnson Deborah (2010) "The undergraduate-postgraduatefaculty triad: unique functions and tensions associated with undergra duate research experiences at research universities" en Journal CBE Life Science Education [En Línea] No. 4. Rockville: American Society for Cell Biology. Disponible en: https://www.ncbi.nlm.nih.gov/pmc/articles/PMC2995772/ [15 de octubre 2018].

Dooley Dian A., Mahon, Mc. Mahon \& Ronan, Oshiro Elizete (2004) “An Undergraduate Research Opportunity: Collaboration Between Undergraduate and Graduate Students" en Journal of Food Science Education [En línea]. No.1, enero. Chicago: Institute of Food Technologists. 
https://onlinelibrary.wiley.com/doi/epdf/10.1111/j.1541-

4329.2004.tb00034.x [25 de enero de 2018].

Espinoza Troconi, María, Cintra Lugones, Ángel \& Pérez Martínez, Lizzete de la Concepción, León Robaina, Rosario (2016) "El proceso de formación científica e investigativa en estudiantes de la carrera de odontología: una mirada desde el contexto venezolano" en Medisan [En línea] http://www.medisan.sld.cu/index.php/san/article/view/769/pdf [12 de febrero de 2019]. No. 6, junio. Santiago de Cuba: Universidad Virtual de Salud. Disponible en:

Flick, Uwe (2007) Introducción a la investigación cualitativa. Madrid: Morata.

Manzon, María (2010) "La comparación de espacios" en Mark, Bray, Mark, Manson \& Bob, Adamson (coordinadores). Educación Comparada.pp. 117-158. Buenos Aires: Granica.

Mertens, Donna (2010) Research and Evaluation in Education and Psychology. Thousand Oaks: SAGE.

Miyahira, Juan (2009) “La investiga ción formativa y la formación pa ra la investigación en el pregrado" en Revista Médica Herediana [En Línea] No. 3, Julio. Lima: Universidad Peruana Cayetano Heredia. Disponible en: http://www.scielo.org.pe/pdf/rmh/v20n3/v20n3e 1.pdf [20 de enero de 2019].

Osada, Jorge, Ruiz-Grosso, Paulo \& Ramos, Mariana (2010) "Estudiantes de pregrado: el Futuro de la investigación" en Revista Peruana de Medicina Experimental y Salud Pública [En Línea] No. 2, junio. Lima: Instituto Nacional de Salud. Disponible en línea en: https://rpmesp.ins.gob.pe/index.php/rpmesp/article/view/1485 [20 de marzo de 2019].

Perdomo, Jhoner \& Valera, Johann (2010) "Análisis de tendencia de los jóvenes investigadores en Venezuela" en Revista de Ciencias Sociales [En Línea] No. 2, junio. Maracaibo: Universidad del Zulia. Disponible en línea en: http://www.redalyc.org/articulo.oa?id=28016298005 [22 de enero de 2019].

Quiñonez, Jeremías \& Velez, Consuelo (2004) "Algunas condiciones pedagógicas para la formación y el desarrollo de la investigación en la universidad" en Revista electrónica Actualidades investigativas en educación [En Línea] No. 1, Enero. San Pedro Montes de Oca: Universidad de Costa Rica. Disponible en: http://www.redalyc.org/pdf/447/44740103.pdf [15 de octubre 2018]

Rodríguez Gómez, Gregorio, Gil Flores, Javier \& García Jiménez, Eduardo (1996) Metodología de la investigación cu alitativa. Malaga: Ediciones Aljibe.

Rojas Betancur, Hector Mauricio \& Méndez Villamizar, Raquel (2017) "Procesos de formación en investigación en la Universidad: ¿Qué les queda a los estudiantes?" en Sophia [En Línea] No. 2, junio. Armenia: Universidad La Gran Colombia. Disponible en línea en: https://www.redalyc.org/pdf/4137/413751844007.pdf [12 noviembre 2019].

Rojas Betancur, Héctor Mauricio (2009) "Formar investigadores e investigadoras en la universidad: optimismo e indiferencia juvenil en temas científicos" en Revista Latinoamericana de Ciencias Sociales Niñez y Juventud [En línea] No. 2, Julio. Manizales: Universidad de Manizales. Disponible en: http://www.scielo.org.co/scielo.php?pid=S1692-

$715 X 2009000300018 \&$ script $=$ sci_abstract\&tlng=es $[15$ de enero de 2018].

Ruíz Herrera, Guillermo Luis, Fernández Montequín, Zoila de la Caridad, \& Horta Muñoz, Dania María (2012) “Estrategia para la formación de habilidades investigativas en estudiantes de medicina" en Ciencias
Médicas [En línea] No. 4, Julio. Pinar del Río: Universidad de Ciencias Médicas de Pinar del Río. Disponible en: https://www.medigraphic.com/pdfs/pinar/rcm-2012/rcm124k.pdf [19 de marzo de 2018]

Torres Soler, Luis Carlos. (2006) "Para qué los Semilleros de investigación" en Reseach Gate [En línea] Colombia: Universidad Nacional de Colombia. Disponible en línea en: https://www.academia.edu/3512546/PARA_QU\%C3\%89_LOS_SEMI LLEROS_DE_INVESTIGACI\%C3\%93N [22 enero 2019].

Viteri, Telmo \& Vázquez, Silvia (2016) "Formación de habilidades de investigación formativa en los estudiantes de la carrera de ingeniería comercial de la Facultad de Ciencias Administrativas de la Universidad de Guayaquil" en Universidad y Sociedad [En Línea] No. 1, Enero. Cienfuegos: Universidad de Cienfuegos. Disponible en: https://rus.ucf.edu.cu/index.php/rus/article/view/302/299 $\quad\left[\begin{array}{ll}15 & \text { de }\end{array}\right.$ febrero de 2018]

Zamora Vera, Narcisa (2014) "La formación investigativa de los estudiantes: Un problema aún por resolver" Escenarios [En línea] No. 2, Julio. Barranquilla: Universidad Autónoma del Caribe. Disponible en línea en: http://ojs.uac.edu.co/index.php/escenarios/article/view/316 $\left[\begin{array}{ll}20 & \mathrm{de}\end{array}\right.$ marzo de 2019].

\section{NOTAS}

\footnotetext{
${ }^{i}$ El registro de datos del Banco Mundial sobre este indicador en México no presenta el correspondiente a 2017.

ii Los profesores de tiempo completo no son de dedicación exclusiva al programa educativo, sino que imparten clases en diferentes carreras y facultades de la universidad. Solo una profesora de tiempo completo pertenece al programa.
} 